\title{
H $\alpha$ SURGE ACTIVITY AT THE FIRST STAGE OF MAGNETIC FLUX EMERGENCE
}

\author{
HIROKI KUROKAWA and GORO KAWAI \\ Kwasan and Hida Observatories, Kyoto University \\ Kamitakara, Gifu 506-13, Japan
}

\begin{abstract}
We present a new observational evidence of the magnetic field reconnection in emerging flux regions (EFR). Our observations show that $\mathrm{H} \alpha$ surges are the first active manifestation of emerging magnetic field in some EFRs. We call them EFR-surges. Examining their morphological and evolutionary characteristics, we conclude that they are produced by the magnetic field reconnection between the EFR and the pre-existing surrounding region.
\end{abstract}

\section{INTRODUCTION}

It is generally known that the emergence of a new active region is recognized by a tiny bright plage and an $\mathrm{H} \alpha$ arch filament system (Waldmier,1937; Bruzek, 1967; Weart and Zirin, 1969; Zwaan, 1985). Surge activity in the first stage of an EFR has received little attention till now. Our observations do reveal, however, that the $\mathrm{H} \alpha$ surges are the very first manifestation of magnetic flux emergence in some EFRs (Kurokawa, 1988). Martin(1983) reported similar evidences. We call them EFR-surges hereafter.

In this paper, we present the morphological and evolutionary characteristics of the EFR-surges, and we discuss them in terms of the magnetic field reconnection.

\section{MORPHOLOGY AND EVOLUTION OF EFR-SURGES}

We have studied the characteristics of the EFR-surges by using the high resolution $\mathrm{H} \alpha$ filtergraph of the $60-\mathrm{cm}$ Domeless Solar Telescope at Hida Observatory, Kyoto University. The results are summarized in the following.

(1) EFR-surge is the first conspicuous activity in some EFRs. Although the very first stage of an EFR is rarely caught, we have some (at least several) observations which show a dark surge with a bright footpoint(tiny plage) at the very beginning of an emerging flux region (Kurokawa,1988; Kurokawa and Kawai, 1993). In these regions, the arch filament system (AFS) appears after the surge activity. 


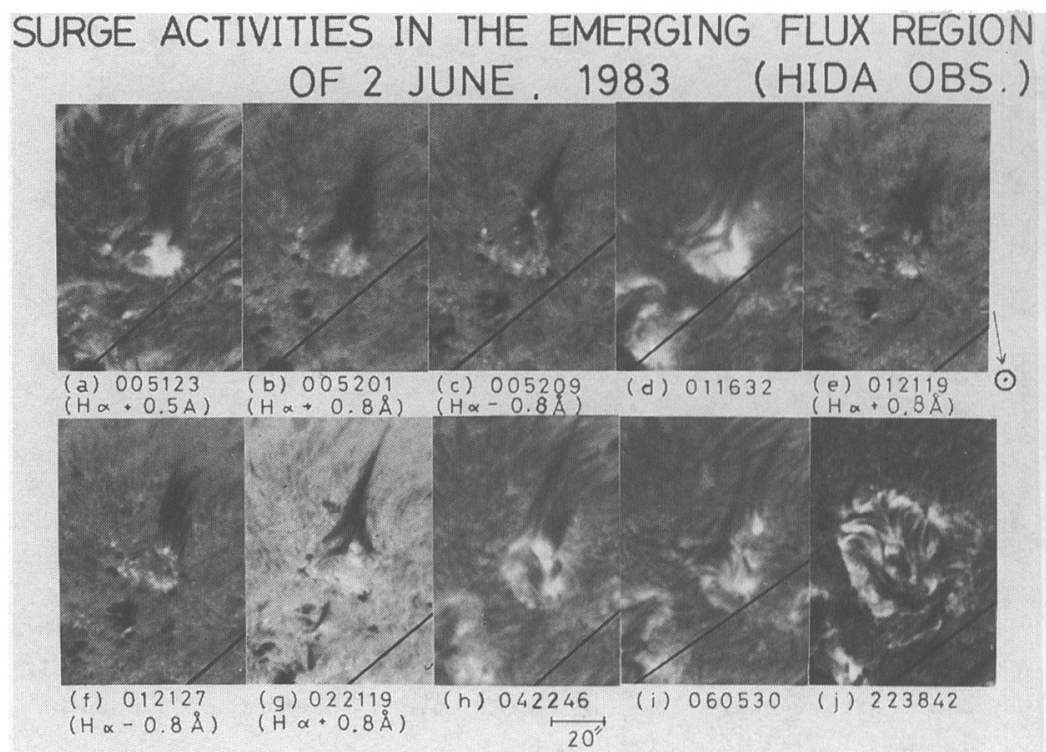

Fig. 1. Evolutional changes of EFR-surges spouting from the EFR of 2 June, 1983. The frames (d), (h), (i) and (j) are taken in $\mathrm{H} \alpha$ line center, the wavelengths used in the other frames are shown below the times of observation (UT). Note in the frame (j) the prorific AFS developments after the surge activities.

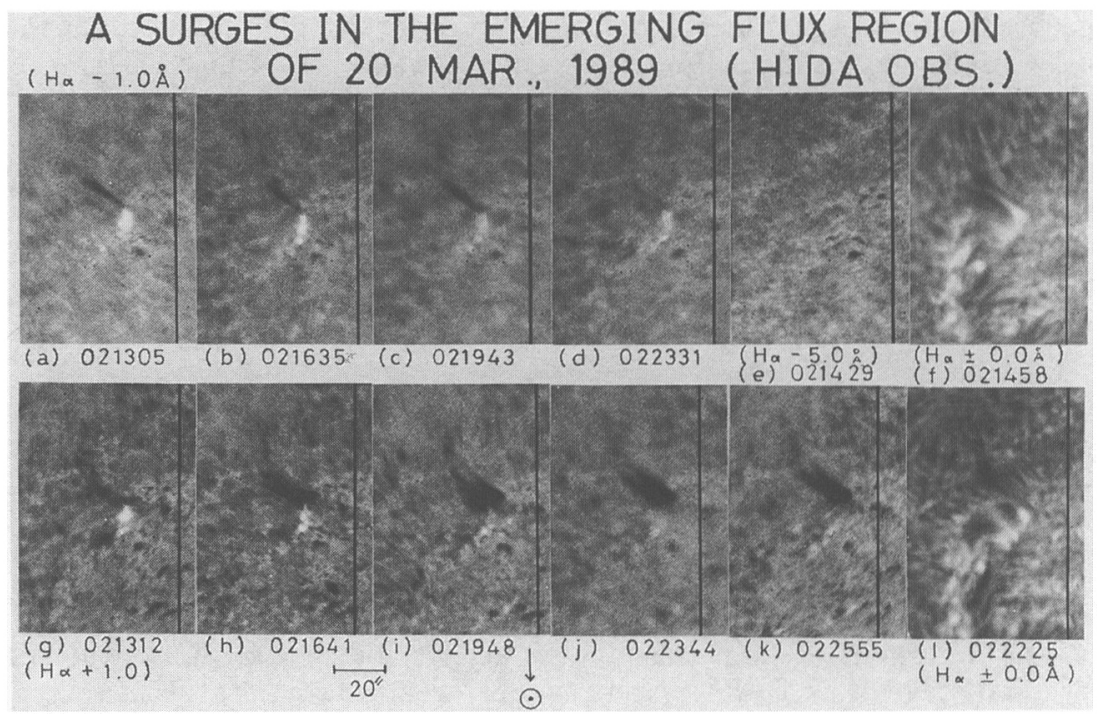

Fig. 2. An EFR-surge spouting from an edge of the AFS of an EFR on 20 March,1989. 
(2) EFR-surges spout out from an edge of the EFR. Even during later stage of an EFR, EFR-surges are often found to spout out from an edge of the EFR where new magnetic flux is still actively emerging and colliding to the surrounding magnetic field. Figure 1 shows a typical example of the EFR-surge obsrved at the birth of active region NOAA4201. Notice that a surge consists of several smaller jets and that the location of the jets shifts outward as the EFR expands (compare the frame (i) with previous frames in Figure 1). At the later stage, many arch filament loops are seen at the location of the previous surge activitiy (see Figure $1(j)$ ). Figure 2 shows another example of a small EFR-surge. The surge is seen at an edge of the arch filament system in the line-center images (Figure 2 (f), (l)).

(3) EFR-surge activities continue for many hours in some EFRs. For the EFR of 1-2 June, 1983, the first surge was obseved about four hours before the time of Figure 1(a) (Kurokawa, 1988). It means that the surge activity continued for more than ten hours in this region. In EFRs observed on 9 October, 1989 and on 2 April, 1989, the surge activities lasted for over five and three hours, respectively (Kurokawa and Kawai, 1993). The lifetime of an individual jet, however, is much shorter or about 10-20 minutes.

(4) EFR-surge often shows a triangular shape, like the Eiffel Tower. Notice the typical triangular shapes of EFR-surges in Figure 1. The characteristic Eiffer-Tower shape is emphasized in the sketch of Figure 3 (a).

(5) EFR-surges show different shapes in the red and blue wings of $\mathrm{H} \alpha$ line throughout their lives. The blue-wing images typically show thin jet-like structures at the upper parts of the EFR-surges, while the red-wing images often show broad skirt-like structures at the lower parts of the EFR-surges (Figure 1 and Figure 2). Consequently EFR-surges cannot be explained by simple rising and falling motions along a single path, and this suggests that the magnetic field configuration of the surge region is changing due to the continuous emergence and reconnection of new magnetic flux, as shown in Figure 3 (b).

(6) EFR-surges spout from the site where emerging magnetic flux collides with pre-existing magnetic field of opposite polarity. These characteristics follow from our examination of the B.B.S.O magnetograms for the EFR of 24 April, 1984 and the Huairou magnetograms for the EFR of 9 0ctober, 1989. Details of these studies will be given in a separate paper (Kurokawa and Kawai, 1993).

\section{SUMMARY AND DISCUSSION}

Our observations reveal that many emerging flux regions show conspicuous $\mathrm{H} \alpha$ surge activity at the earliest stages of their emergences. A dark surge is found with its bright footpoint to be the first manifestation of new magnetic flux in some EFRs. In these regions, the AFS is formed after the surge activity. Even during later stage of an EFR, EFR-surges are often found to spout out from an edge of the EFR where the magnetic polarity of the EFR is opposite to that of the surrounding region. We call them EFR-surges.

The morphological and evolutionary characteristics of the EFR-surges, strongly suggest that they are produced by magnetic field reconnection between the EFR and the pre-existing surrounding region. We give a schematic 
model of the reconnection and the EFR-surge formation in Figure 3(b), where thick solid, broken and thin solid lines represent the emerging, pre-existing and reconnected magnetic field lines, respectively. The shaded parts in Figure 3(b) show the EFR-surges produced by the magnetic field reconnection. Notice a morphological agreement between the observed and the modeled EFR-surges, comparing schematic pictures (a) and (b) in Figure 3.

It is interesting to compare our findings with the two-dimensional magnetohydrodynamic numerical simulation of Shibata et al.(1992), who found that the cool jet is accelerated along the current sheet up to the speed of $50-100 \mathrm{kms}^{-1}$ by magnetic field reconnection between an emerging flux and overlying coronal magnetic field.

Finally, we stress the importance of detailed magnetic field measurements of more EFRs to confirm our reconnection model of EFR-surges. High resolution magnetograms are needed, because EFR-surges consist of many smaller jets, which suggests the emergence of many small magnetic bipoles in one EFR.
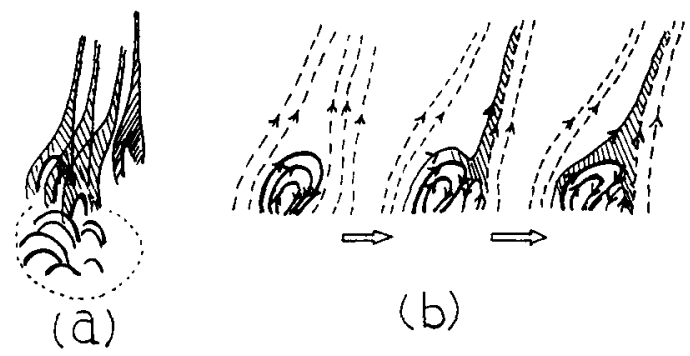

Fig. 3. (a) Sketch of the EFR-surges observed in the 2-June-1983 EFR shown in Figure 1. (b) Schematic model of the magnetic field reconnection and the EFR-surge formation.

Our thanks are due to Prof. H. Zirin and Dr. Ai Guoxiang for their encouraging us to study the B.B.S.O. and Huairou magnetograms, respectively. We are grateful to Prof. C. Zwaan for his helpful comments in revising the manuscript. We also wish to thank Drs. Y. Nakai, Y.Funakoshi and R. Kitai for their helps in observations, and Dr. K. Shibata for his discussion on the reconnection model of the EFR-surges.

\section{REFERENCES}

Bruzeck, A.: 1967, Solar Physics 2451.

Kurokawa, H.: 1988, Vistas in Astronomy 3167.

Kurokawa, H. and Kawai, G.: 1993, Solar Physics, to be published.

Martin, S.F.: 1983, Bull. American Astron. Soc. 15971.

Shibata, K., Nozawa, S., and Matsumoto, R.: 1992, Publ. Astron. Soc. Japan 44265.

Waldmier, M: 1937, Zs. f. Ap. 1491.

Weart, S. and Zirin, H.: 1969, Publ. A.S.P. 81480.

Zwaan, C.: 1985, Solar Physics 100397. 